\title{
FIRST MEASUREMENTS OF THE ION ENERGY DISTRIBUTION AT THE DIVERTOR STRIKE POINT DURING DIII-D DISRUPTIONS
}

\author{
by \\ P.B. PARKS, R. BASTASZ, D. WHYTE, \\ N.H. BROOKS, W.R. WAMPLER, W.P. WEST, \\ and C.P.C. WONG
}

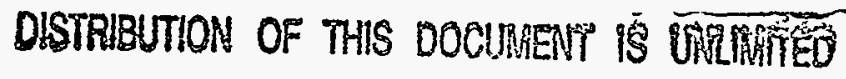

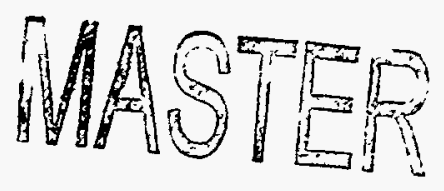

DECEMBER 1995 


\section{DISCLAIMER}

This report was prepared as an account of work sponsored by an agency of the United States Government. Neither the United States Government nor any agency thereof, nor any of their employees, makes any warranty, express or implied, or assumes any legal liability or responsibility for the accuracy, completeness, or usefulness of any information, apparatus, product, or process disclosed, or represents that its use would not infringe privately owned rights. Reference herein to any specific commercial product, process, or service by trade name, trademark, manufacturer, or otherwise, does not necessarily constitute or imply its endorsement, recommendation, or favoring by the United States Government or any agency thereof. The views and opinions of authors expressed herein do not necessarily state or reflect those of the United States Government or any agency thereof. 


\title{
FIRST MEASUREMENTS OF THE ION ENERGY DISTRIBUTION AT THE DIVERTOR STRIKE POINT DURING DIII-D DISRUPTIONS
}

\author{
by \\ P. B. PARKS, R. BASTASZ, * D. WHYTE, $\dagger$ \\ N.H. BROOKS, W.R. WAMPLER, W.P. WEST, \\ and C.P.C. WONG
}

This is a preprint of a paper presented at the 16th IEEE/NPSS Symposium on Fusion Engineering, September 30-October 5, 1995, Champaign, Illinois, and to be printed in the Proceedings.

\author{
Work supported by \\ U.S. Department of Energy \\ Contract DE-AC03-89ER51114
}

*Sandia National Laboratories

$\dagger$ INRS-Energie et Materiaux

GENERAL ATOMICS PROJECT 3466

DECEMBER 1995 


\section{DISCLAMTISR}

Portions of this document may be illegible in electronic:image products. Images are produced from the best available original document. 


\title{
First Measurements of the Ion Energy Distribution at the Divertor Strike Point During DIII-D Disruptions*
}

\author{
P.B. Parks, R. Bastasz, † D. Whyte, $\ddagger$ N.H. Brooks, W.R. Wampler, $†$ W.P. West, and C.P.C. Wong \\ General Atomics, P.O. Box 85608, San Diego, California 92186-9784 \\ TSandia National Laboratories \\ $\neq$ INRS-Energie et Materiaux
}

\begin{abstract}
Plasma/wall interaction studies are being carried out using the Divertor Materials Exposure System (DiMES) on DIII-D. The objective of the experiment is to determine the kinetic energy and flux of deuterium ions reaching the divertor target during argon-induced radiative disruptions. The experiment utilizes a special slotted ion analyzer mounted over a Si sample to collect the fast charge-exchange (CX) deuterium neutrals emitted within the recycled cold neutral layer (CNL) which serves as a CX target for the incident ions.

A theoretical interpretation of the experiment reveals a strong "forward" pitch-angle dependence in the approaching ion distribution function. The depth distribution of the trapped $D$ in the Si sample was measured using low-energy direct recoil spectroscopy. Comparison with the TRIM code using monoenergetic ions indicated that the best fit to the data was obtained for an ion energy of $100 \mathrm{eV}$. An estimate of the CNL thickness $\int \mathrm{nd} \ell$ indicates that during disruptions the CNL "cushion" is thick enough to reduce the local ion heat load by $\sim 30 \%$ due to $\mathrm{CX}$ refluxing.
\end{abstract}

\section{INTRODUCTION}

Plasma disruptions are a serious concern in tokamak design because of the high impulsive heat loads which can cause strong erosion of divertor materials due to enhanced sputtering, or melting/ablation in the most severe cases. Predictions of net erosion rates and hence component lifetimes are very difficult and are highly dependent on the plasma conditions over the divertor target. It is therefore necessary to characterize the properties of the scrape-off plasma near the divertor target plate under these special conditions. The angular and energy distribution of ions impinging on the divertor plate is. needed in order to calculate and benchmark surface erosion, in plasma transport, and redeposition of impurity atoms/ions due to sputtering [1,2]. The ion distribution near the target plate also controls the thickness of the vapor shield [3] and the erosion rate.

DIII-D is an ideal experimental platform for studying disruption issues because the plasma can be operated in a singlenull configuration similar to ITER [4]. Plasma wall interaction studies are being carried out in the DiMES program. One primary experimental need is to provide information on the angular and energy distribution of the incident ions and CX neutral atoms on the divertor plate during a disruption.
A special slotted ion analyzer able to withstand high-heat flux was designed for characterizing the ions/CX neutrals impinging on the divertor target near the outboard divertor strike point. The device actually collects fast $\mathrm{CX}$ neutrals originating from ions interacting with the cold neutral layer (CNL) over the divertor surface.

First results from the slotted ion analyzer and theoretical interpretation are reported here. In Section II, we describe the apparatus, and present measurements of the fluxes and energies of the particles collected during a series of Ar-induced disruptions. In Section III we employ a simple kinetic model to establish the necessary relationship between the directional flux of the collected neutrals and the gyro-motion of the approaching ions. In Section IV we formulate an expression for the total neutral particle flux or areal density at the bottom of the slot. Consideration is given to the actual slot geometry and limited solid angle of collection. The measured and calculated areal densities of the implanted D atoms are then compared in Section V.

\section{THE EXPERIMENTAL APPARATUS AND RESULTS}

The slotted particle analyzer consists of a silicon crystal wafer, the collector, covered by a slotted mask, which provides four viewing angles into the divertor plasma at $\beta=$ $0,30,45$, and 60 degrees. The entire assembly is mounted on the retractable DiMES head as shown in Fig. 1. The lengthwise direction of the slots, with length $\ell=1.21 \mathrm{~cm}$, is perpendicular to the magnetic field, which makes an oblique angle $\alpha=2.7$ degree with respect to the divertor target plate. The entrance slit at the top of the assembly is flush mounted with the target plate, and it is shaped and positioned relative to the bottom slots as if the walls of these "stubby" slots were individually extended all the way up to the entrance hole as shown in Fig. 2. The slot half-width is $\delta=0.5 \mathrm{~mm}$, and the slot depth (from entrance plane to $\mathrm{Si}$ crystal), is $\mathrm{d}=$ $0.596 \mathrm{~cm}$. This canted slot arrangement serves as a directional analyzer for the approaching ions which leave a CX neutral "imprint" at each view angle; the limited amount of fast neutrals striking the Si sample prevents damage.

The Si sample was exposed to three consecutive Ar-induced disruptions: DIII-D shots 81166-8. In-shot measured plasma parameters such as heat-flux on the divertor target plate were determined from high-speed infrared thermography. The actual value on the sample is $Q_{\perp}=70 \mathrm{MW} / \mathrm{m}^{2}$. The temporal behavior indicates a disruption pulse width of $t_{d} \sim 2 \mathrm{~ms}$.

\footnotetext{
*Work supported by the U.S. Department of Energy under Contract No. DE-AC03-89ER51114.
} 


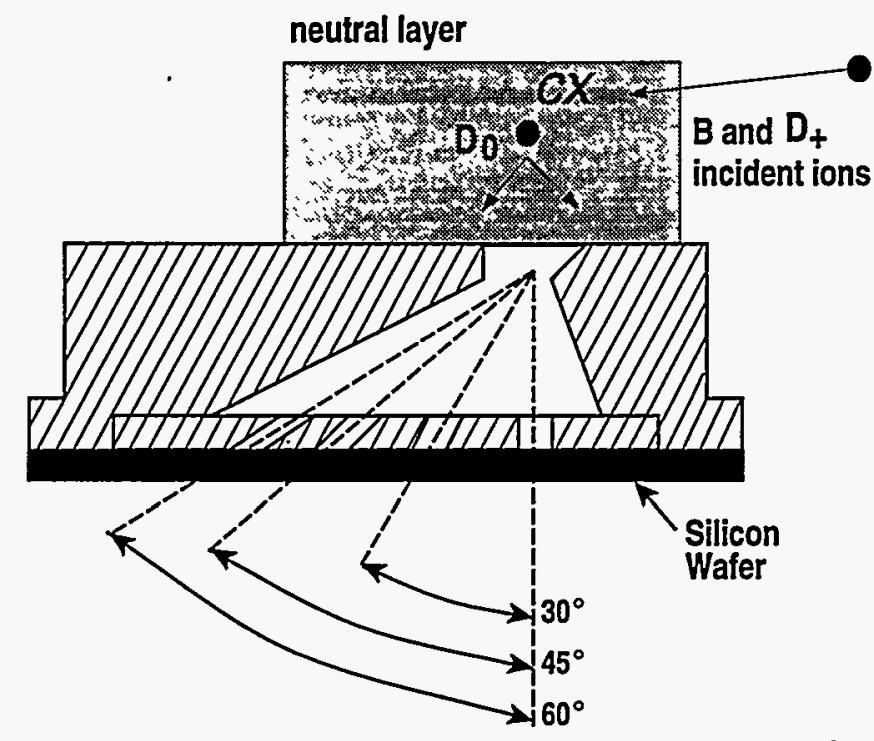

Fig. 1. Slotted ion anlyzer mounted on DiMES probe in DIII-D divertor

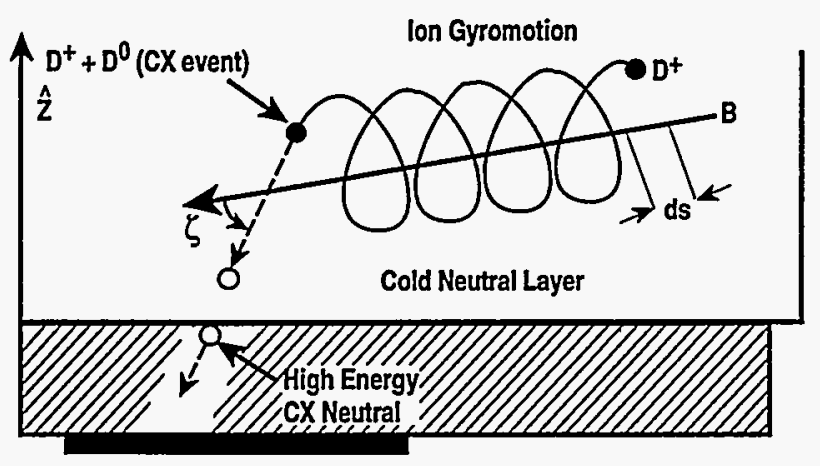

Fig. 2. Ion gyro motion in cold neutral layer above DiMES sample

The amount of $\mathrm{D}$ trapped in the Si was measured using the $\mathrm{D}\left({ }^{3} \mathrm{He}, \mathrm{p}\right){ }^{4} \mathrm{He}$ nuclear reaction to quantitatively determine the areal density $\mathrm{N}_{\mathrm{A}}\left(\mathrm{m}^{-2}\right)$ of $\mathrm{D}$ within the first few $\mu \mathrm{m}$ of the sample. The amounts are displayed in Fig. 3 as $\beta$ is varied.

The trapped D depth distributionwas measured at each view angle using low-energy direct recoil spectroscopy. A $1.00 \mathrm{keV} \mathrm{Ne}{ }^{+}$beam served both for the $D$ analysis and for sputtering craters in the sample. An energy analyzer recorded the intensity of positive ions emitted near $0.27 \mathrm{keV}$. For each profile, the signal was integrated for a constant incremental $\mathrm{Ne}^{+}$dose and then recorded for 200 successive intervals. The sputter erosion rate was determined by measuring the final crater depths with a profilometer. Depth profiles were generated by converting the dose scale to depth, and the recoil signal to D concentration. The depth conversion was made using an erosion rate derived from the profilometry measurements.

A range curve calculated for monoenergetic $D$ in Si based on Magee's experimental measurements [6] finds that the indicated energy for D striking the sample is approximately $100 \mathrm{eV}$ at the normal view angle. The incident particle energy appears to be slightly lower at the other view

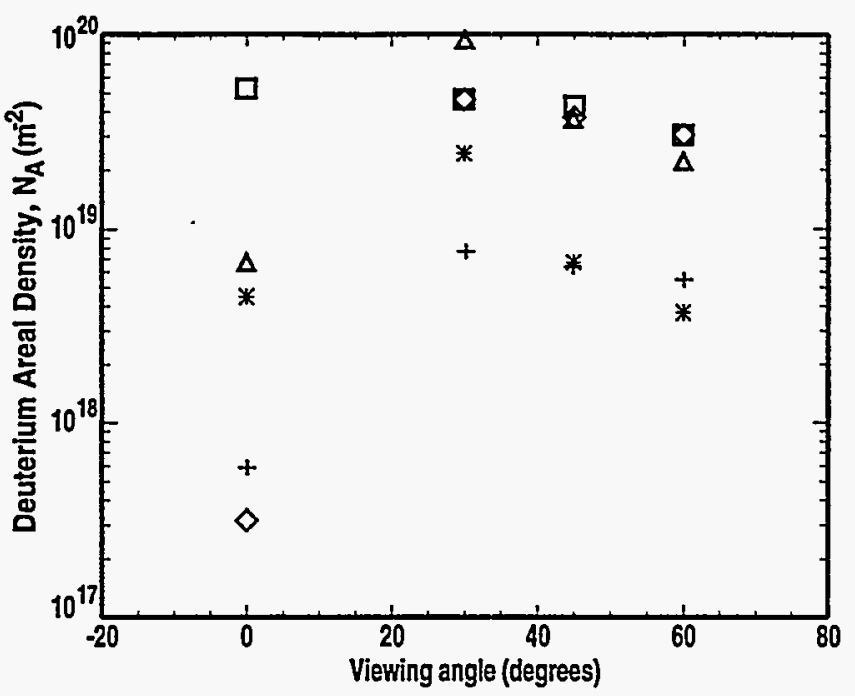

Fig. 3. Measured areal density of $D$ neutrals collected during disruption and a comparison with theoretical model ( $\square$ experiment; $\diamond$ theory, $\tau=$ $0.7, k=1.45 ; \Delta$ theory, $\tau=0.7, k=0 ;+$ theory, $\tau=0.085, k=2$; * theory, $\tau=0.085, \mathrm{k}=0$.

angles. A number of implant distributions were calculated using the TRIM code $[7,8]$. The calculations were restricted to energies $\geq 100 \mathrm{eV}$, a region where the interatomic potential is considered reliable. The implant profile from TRIM was compared with the data for normal $(\beta=0)$ incidence, verifying that $E_{0} \approx 100 \mathrm{eV}$.

From these results we can estimate the density of the ions at the target plate. Combine the heat flux formula on the divertor target, $Q_{\perp}=\sin \alpha \Gamma_{\| i} \delta_{H} T_{i}$, with Emmert et al.'s expression for the flux of collisionless ions at a sheath edge $\Gamma_{\| i}=1 / 4 n_{i} v_{i} \beta_{E}\left(\beta_{E}=2.2\right)$ [5]. Taking $T_{i}=100 \mathrm{eV}$, and heat flux transmission coefficient $\delta_{H}=5$, we find $n_{i}=$ $3 \times 10^{14} \mathrm{~cm}^{-3}$. Our justification for assuming collisionless approaching ions is that the ions had their last collision at a distance from the target plate $\sim \sin \alpha \lambda_{\mathrm{ii}}=3.6 \mathrm{~cm}$. This exceeds the neutral density scale length, which is deduced in the next section to be approximately $\sim 1 \mathrm{~cm}$.

\section{ON THE RELATION BETWEEN THE INCIDENT IONS AND THE FAST CX NEUTRALS REACHING DIMES SAMPLE}

To characterize the $\mathrm{CNL}$ and the ion distribution function of the approaching (fast) ions we need to see how they are related to the measured quantities, namely the areal density of the implanted D atoms, or the fast neutral atom flux at the slot bottoms, and its variation with view angle $\beta$.

Referring to Fig. 2 again, ions having different pitch angle-like variables $\cos \xi \equiv \mu=v_{\|} / v$ with respect to the magnetic field have different path-lengths through the $\mathrm{CNL}$, and therefore suffer different amounts of CX attenuation. Assuming a one dimensional z-variation normal to the target plate, the kinetic equation for the attenuated fast ion distribution $f(v, \mu, z)$, is given by

$$
v \mu \sin \alpha \partial f / \partial z=-X(f, g)+v_{\text {ion }} g
$$


where $g$ is the neutral atom distribution function, $X(f, g)$ is the charge exchange collision operator [9], and $v_{\text {ion }}$ is the electron impact ionization frequency (neglected here). We can simplify $\mathrm{X}(\mathrm{f}, \mathrm{g})$ by neglecting fast ion $\mathrm{CX}$ with the hot(h) neutral component relative to $\mathrm{CX}$ with the cold(c) neutral component, which we represent by a delta function in velocity. This yields $X(f, g) \cong n_{c}(z) v \sigma_{x}(v) f(v, \mu, z)$, where $\sigma_{\mathrm{X}}(\mathrm{v})$ is the $\mathrm{CX}$ cross section. This separation can be motivated under disruption conditions because the ratio of the respective layer thicknesses $\int n_{c} d z / n_{h} d z>$ $\left(T_{i} / T_{n c}\right)^{1 / 2}\left(L_{c} L_{h}\right) \gg 1$. The first inequality is related to the fact that fast CX neutral reflux back to plasma can be no larger than the efflux of cold neutrals emitted from the target plate. To achieve the second equality, we observe that $T_{1} \approx 100 \mathrm{eV}$ is much larger than the cold neutral Franck-Condon temperature $T_{n c} \approx 2-3 \mathrm{eV}$, and the respective density scale lengths are comparable. (A random walk argument [9] shows that outside the CNL the hot density scale length $L_{h} \sim\left(\lambda_{\text {ion }} \lambda_{\mathrm{f}}\right)^{1 / 2}=0.95 \mathrm{~cm}$, where the respective mean free paths for ionization and $\mathrm{CX}$ are $\lambda_{\text {ion }}=$ $\mathrm{v} / \lambda_{\text {ion }}=1.1 \mathrm{~cm}$ and $\lambda_{\mathrm{x}}=1 / \mathrm{n}_{\mathrm{i}} \sigma_{\mathrm{x}}=0.83 \mathrm{~cm}$. It will be shown that the CNL scale length is $L_{c} \sim 1 \mathrm{~cm}$, so indeed $\mathrm{L}_{\mathrm{c}} \sim \mathrm{L}_{\mathrm{h}}$ )

We examine the solution when the CNL has an exponential density profile, $n_{c}=n_{c 0} \exp -(z-d) / L_{c}$ where $n_{c 0}$ is the neutral density at the target plate $z=d$. This is simply

$$
\begin{aligned}
f(v, \mu, z) & =f_{\infty}(v, \mu) \exp \\
& \times\left(\frac{-\sigma_{x}(v) n_{c o} L_{c} e^{-(z-d) / L_{c}}}{\mu \sin \alpha}\right)
\end{aligned}
$$

The approaching ion distribution function is chosen to have a simple form,

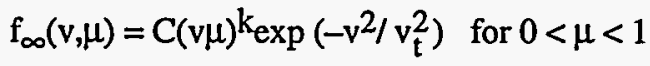

$$
\begin{aligned}
& =0 \text { for }-1<\mu<0
\end{aligned}
$$

where the constant $C$ can be normalized to the ion density $\mathrm{n}_{\mathrm{i}}$ obtained in Section $\mathrm{I}$, and for continuity the pitch angle anisotrophy index $k>0$; it's value will be adjusted to match the $\mathrm{N}_{A}(\beta)$ data. In essence, the ions are the attracted species in the pre-sheath, so negative values of $v_{\|}$are not allowed. For $\mathrm{k}=2$, this form takes after the anisotropic collisionless ion model of Emmert et al. [5], and for $k=0$ the approaching ions would be isotropic.

Turning to the fast CX neutral transport, we employ a spherical coordinate system with polar axis in the z-direction, and origin affixed to the center of the slot bottom $(r=0)$. In Fig. 4 is shown a side view of a canted slot in the $\phi=\pi / 2$ plane of symmetry. We derive an expression for the total neutral flux at the origin. The kinetic equation for the fast $\mathrm{CX}$ neutrals going towards the origin is given by

$$
\mathrm{v} \hat{\Omega} \cdot \nabla \mathrm{g}=\mathrm{X}(\mathrm{f}, \mathrm{g})-\lambda_{\text {ion }} \mathrm{g}
$$

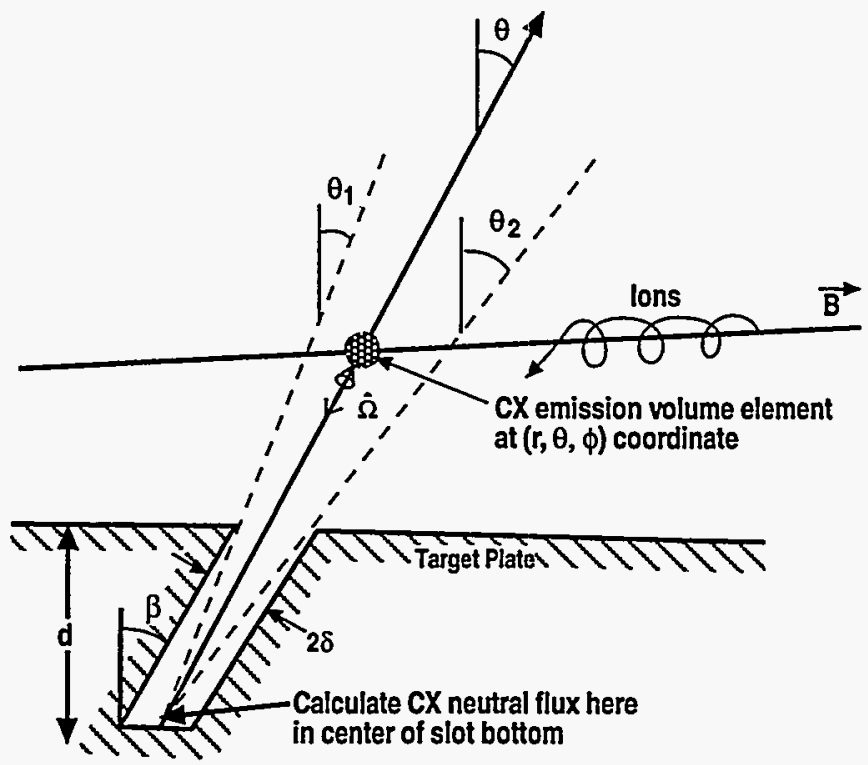

Fig. 4 Cross sectional slot geometry, and spherical coordinate system used in the calculation of fast neutral flux to center of slot bottom (origin).

where $\hat{\Omega}=-\hat{\mathbf{r}}$ is the unit normal directed towards the origin. Fast CX neutrals emitted from the volume element at $(\theta, \phi, \Gamma)$ in direction $\hat{\Omega}$ come from ions with pitch angle given by the geometric relation $\mu=\sin \alpha \cos \theta+\cos \alpha \sin \theta$ $\sin \phi$. Integration of Eq. (4) along the radial coordinate from $r=\infty$ to $r=0$, yields the directional flux $\left(I_{n}=v g\right)$ of the fast neutrals at the origin: $I_{n s}(v, \theta, \phi)=G[v, \mu(\theta, \phi)] /$ $\cos \theta$, where $G[v, \mu(\theta, \phi)]=\mu v \sin \alpha f_{\infty}(v, \mu)[1-\exp -\tau / \mu]$, and $\tau=\sigma_{\mathrm{x}}(\mathrm{v}) \mathrm{n}_{\mathrm{c} 0} L_{d} / \sin \alpha$ is a dimensionless CNL thickness.

The total neutral flux at the silicon sample with speeds between $v$ and $v+d v$ is therefore

$$
\Gamma_{n s}(v)=\int G(v, \theta, \phi) \sin \theta d \theta d \phi
$$

Finally, the areal density of implanted neutrals in the sample is $N_{A}=\iint \Gamma_{n s}(v) v^{2} d v d t$. Since $\sigma_{x}(v)$ is weakly varying for ion speeds corresponding to $100 \mathrm{eV}$, we can set $\sigma_{\mathrm{x}}=$ $4 \times 10^{-15} \mathrm{~cm}^{-2}$, allowing a straightforward integration over speed. The time integration is taken as $\int d t \sim t_{d}$.

\section{VIEW FACTOR CALCULATION}

The slot walls limit the solid angle view, or $\theta, \phi$ integration limits, making evaluation of the double integral in Eq. (5) quite complicated. Fortunately, one can do a single integration in $\mu$ while trying out different forms of $f_{\infty}(v$, $\mu$ ) until the computed areal density in each slot is matched to the data. For example, consider flux to the $\beta \neq 0$ canted slots. There are three "critical" polar angles; the first two, $\theta_{1}$ and $\theta_{2}$ are indicated in Fig. 4, and the maximum polar angle is given by $\tan \theta_{\max }=\left(\ell^{2} / 4 \mathrm{~d}^{2}+\tan ^{2} \beta\right)^{1 / 2}$. By symmetry we need only consider the $\theta$ integration over theinterval $-\pi / 2 \leq \phi \leq \pi / 2$. Within this range, the minimum 
value of $\phi$ for a given polar angle $\phi$ is given by $\sin \phi_{\min }(\theta)$ $=\tan \theta_{1} \cot \theta$. The maximum value of $\phi$ is $\pi / 2$ when $\theta$ is less than $\theta_{2}$, otherwise it is given by $\sin \phi_{\max }(\theta)=$ $\tan \theta_{2} \cot \theta$. Thus Eq. (5) becomes

$$
\begin{aligned}
\Gamma_{\mathrm{ns}}(\mathrm{v}) & =2 \int_{\theta_{1}}^{\theta_{2}} \sin \theta d \theta \int_{\phi_{\min }(\theta)}^{\pi / 2} G(v, \theta, \phi) d \phi \\
& +2 \int_{\theta_{2}}^{\theta_{\max } \sin \theta d \theta} \int_{\phi_{\min }(\theta)}^{\phi_{\max }(\theta)} \mathrm{G}(\mathrm{v}, \theta, \phi) d \phi
\end{aligned}
$$

We next switch integration variables, using the identity $\mathrm{d} \theta \mathrm{d} \phi=\mathrm{J}^{-1} \mathrm{~d} \theta \mathrm{d} \mu$, where the transformation Jacobian is given by $\mathrm{J}(\theta, \mu)=\left(\cos ^{2} \alpha-\cos ^{2} \theta-\mu^{2}+2 \mu \sin \alpha \cos \theta\right)^{1 / 2}$. Reversing the order of integration allows the $\theta$-integration, now with $\mu$-dependent limits, to be done analytically. After considerable manipulation, the flux integral reduces to

$$
\begin{aligned}
& \Gamma_{\mathrm{ns}}(v)=\dot{\pi} \int_{\sin \left(\alpha+\theta_{1}\right)}^{\sin \left(\alpha+\theta_{2}\right)} \mathrm{G}(\mu, v) \mathrm{d} \mu \\
& +2 \int_{\alpha_{t} \sin \alpha \cos \theta_{\max }}^{\sin \left(\alpha+\theta_{1}\right)} G(\mu, v) \sin ^{-1}\left[\frac{\mu\left(\cot \alpha-\tan \theta_{1}\right)}{\alpha_{t}\left(1-\mu^{2}\right)^{1 / 2}}\right] d \mu \\
& -2 \int_{\alpha_{\mathrm{t}} \sin \alpha \cos \theta_{\max }}^{\alpha_{\mathrm{w}} \sin \alpha \cos \theta_{\max }} \mathrm{G}(\mu, \mathrm{v}) \sin -1\left[\frac{\cos \theta_{\max }-\mu \sin \alpha}{\cos \alpha\left(1-\mu^{2}\right)^{1 / 2}}\right] \mathrm{d} \mu \\
& -2 \int_{\alpha_{w} \sin \alpha \cos \theta_{\max }}^{\sin \left(\alpha+\theta_{2}\right)} \mathrm{G}(\mu, v) \sin -1\left[\frac{\mu\left(\cot \alpha-\tan \theta_{2}\right)}{\alpha_{w}\left(1-\mu^{2}\right)^{1 / 2}}\right] \mathrm{d} \mu
\end{aligned}
$$

where $\alpha_{\mathrm{t}}=1+\tan \theta_{1} \cot \alpha$, and $\alpha_{\mathrm{w}}=1+\tan \theta_{2} \cot \alpha$. The expression for the $\beta=0$ slot is similar; the difference is associated with the condition in Eq. (3) that negative values of $\mu$ are not allowed.

\section{SUMMARY}

The results of the flux calculation are summarized in Fig. 3. In order to fit the experimental $N_{A}(\beta)$ data we had to adjust two quantities: the neutral layer thickness $\tau$, which affects the magnitude and shape of the curves; and the ion anisotrophy parameter $k$, which affects mostly the shape. The best fit to the data $\beta \neq 0$ indicates that $\tau=0.70$, and $\mathrm{k}=1.45$. This means that $\mathrm{n}_{\mathrm{co}} \mathrm{L}_{\mathrm{c}} \approx 2 \times 10^{13} \mathrm{~cm}^{-2}$, and e-folding length $\mathrm{L}_{c} \approx 1 \mathrm{~cm}$. The angular ion distribution function is anisotropic, and is near 2, as suggested by the collisionless ion pre-sheath model of Emmert et al. [5]. Unfortunately, the measured $\mathrm{N}_{\mathrm{A}}$ for 0 degree slot is about a factor of 10 larger than the model result. This discrep- ancy may be due to the accumulation of CX neutrals in the 0 degree slot from the core plasma between disruptions.

To gain an appreciation for the apparently enhanced CNL thickness during a DIII-D disruption, we have plotted in Fig. 5 the theoretical hot particle transmission coefficient $\mathrm{T}(\tau)$ defined as:

$$
\mathrm{T}=\frac{\left(\Gamma_{\text {Neutral }}+\Gamma_{\text {Ions }}\right)_{\text {Plate }}}{\left(\Gamma_{\text {Ions }}\right)_{\text {Incident }}}
$$

This result indicates that during the disruption experiment, with $\tau=0.70, T=0.7$, the CNL can help shield the target surface from the ion heat flux by refluxing fast $\mathrm{CX}$ neutrals back to plasma.

Further measurements and theoretical modeling are needed before definitive statements can be made concering the scaling of the CNL thickness, and the ion distribution function with target heat flux $Q_{\perp}$.

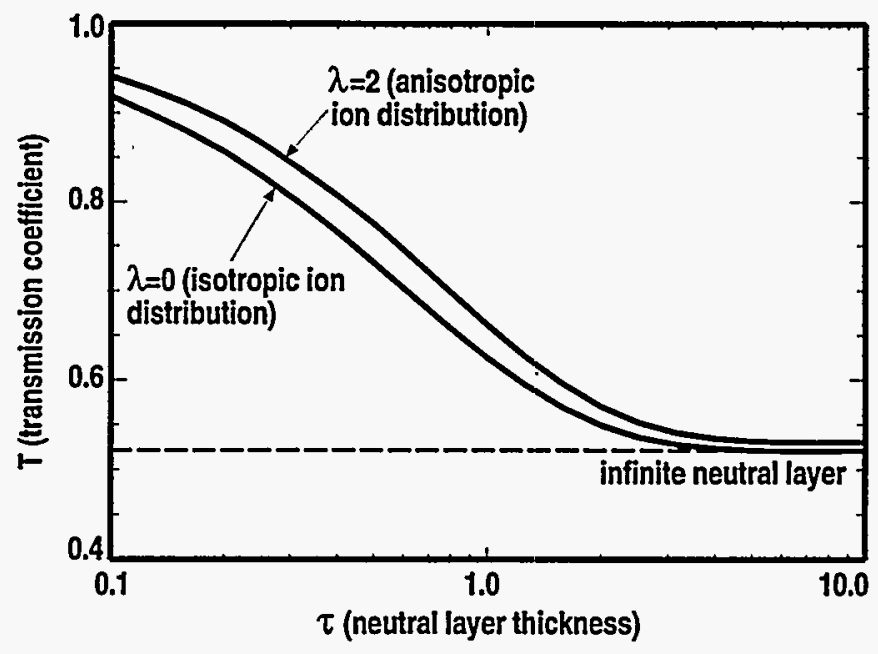

Fig. 5. Flux transmission factor through cold neutral layer as a function of its thickness $t$ defined in text.

\section{REFERENCES}

[1] J.N. Brooks, Nucl. Technol. Fusion 4, p. 3, 1983.

[2] J.N. Brooks, "Divertor erosion study for TPX and implications for steady-state fusion reactors," Proc. 16th IEEE Symp. on Fusion Energy, Champaign, Illinois, to be published.

[3] J. Gilligan, "Effect of disruptions on plasma-facing components," Proc. 16th IEEE Symp. on Fusion Energy, Champaign, Illinois, to be published.

[4] ITER Conceptual Design: Interim Report (IAEA,Vienna,1990), Report No. IAEA-ITER-B5-7.

[5] G.A. Emmert, R.M. Wieland, A.T. Hense, J.N. Davidson, "Electric sheath and pre-sheath in a collisionless, finite ion temperature plasma," Phys. Fluids 23, p. 803, 1980.

[6] C.W. Magee, S.A. Cohen, D.E. Voss, and D.K. Brice, "Hydrogen profiling in silicon using secondary ion mass spectroscopy," $\mathrm{NuCl}$. Instrum. Methods 168, p. 383, 1980.

[7] J.P. Biersak, L.G. Haggmark, "A Monte Carlo code for computing trajectoru ranges in matter," Nucl. Instrum. Methods 174, p. 257, 1980.

[8] J.F. Ziegler, J.P. Biersak, U. Littmark, The Stopping and Range of Ions in Solids, vol. 1, New York: Pergamon, 1985, p. 321.

[9] R.D. Hazeltine, M.D. Calvin, P.M. Valanju, E.R. Solano, "Analytical calculation of neutral transport and its effect on ions," Nucl. Fusion 32, p. 3, 1992. 